\title{
Intelligent Content-Centric Networking Routing Strategy: A Bacterial Quorum Inspired Pattern
}

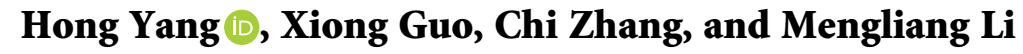

China Electronics Standardization Institute, Beijing 100007, China

Correspondence should be addressed to Hong Yang; 13810019818@163.com

Received 8 January 2021; Revised 9 March 2021; Accepted 13 May 2021; Published 2 June 2021

Academic Editor: Wei Wang

Copyright (C) 2021 Hong Yang et al. This is an open access article distributed under the Creative Commons Attribution License, which permits unrestricted use, distribution, and reproduction in any medium, provided the original work is properly cited.

In recent years, the Content-Centric Networking (CCN) has attracted much attention from the global Internet experts; in particular, it has demonstrated the outstanding effect on the application in the field of Internet of Things (IoT). At present, the routing technique of ICN is subjected to the dynamic change of network environment with the development of mobile Internet. Therefore, this paper proposes an Intelligent CCN routing strategy based on Bacterial Quorum pattern (ICBQ). The ICBQ tries to simulate the behaviors of bacteria, including quorum sensing and adaptive chemotaxis. Meanwhile, the quorum sensing can obtain the parameter information on bandwidth, delay, and error rate to facilitate the subsequent forwarding of packets. The adaptive chemotaxis can select the optimal interface to forward the packets through the information measurement. The simulation is driven based on the real Netflix dataset over the GTS network topology, and the experimental results show that the proposed ICBQ has better performance in terms of routing success rate, routing delay, load balance, and energy efficiency.

\section{Introduction}

With the rapid development of Internet of Things (IoT) [1], cloud computing [2], and mobile Internet [3], it is very difficult for the current Internet to support the efficient content transmission, since there have been a large number of mobile devices generating the data traffic. For example, according to the report from Cisco, the global IP traffic is expected to reach $3.3 \mathrm{ZB}$ by 2021 [4]. Under such context, it is very necessary to pay attention to the design of the future Internet. To the best of our knowledge, the Content-Centric Networking (CCN) [5] is the most popular future Internet paradigm, which can greatly address these issues, for example, content distribution, mobility, and security. It is worth noting that the CCN is the considerably significant networking system architecture, and two special international conferences from the well-known academic organizations, that is, IEEE HotICN and ACM ICN, were held for each year from 2018 and 2014, respectively. Regarding the research fields, $\mathrm{CCN}$ includes three main aspects, that is, naming, routing, and caching. Among them, the routing plays an important role, which performs the retrieval of contents via the forwarding of interest packets.

However, as mentioned above, the number of mobile devices has been increasing sharply and thus the network status cannot be obtained conveniently, which causes the fact that the traditional CCN routing strategies cannot realize the efficient content retrieval. Thus, the intelligent CCN routing technique has attracted the great attention from the academia and industry. At present, there have been some proposals on intelligent CCN routing. For example, in [6], a particle swarm optimization based CCN routing was proposed. It used the forwarding experiences of particles to maintain the forwarding probability of entry with the multiple Quality of Service (QoS) constraints consideration. In [7], an energy-efficient QoS routing was devised, in which a multiple-objective decision problem was involved. It used the intelligent drops algorithm to address the NP-hard problem. In [8], an intelligent Q-learning based reinforcement learning scheme was proposed to address the congestion control issue in the $\mathrm{CCN}$ routing. It used Q-learning 
method to learn the network status and designed a hop-byhop forwarding mode according to the learning value. In [9], an intelligent ant colony optimization inspired $\mathrm{CCN}$ routing strategy was introduced, which simulated the foraging behaviors of ants to obtain the closest contents via the forwarding of interest ants.

Different from the previous intelligent routing proposals, we in this work propose an intelligent $\mathrm{CCN}$ routing based on Bacterial Quorum pattern (ICBQ). The ICBQ simulates the behaviors of bacteria, including quorum sensing and adaptive chemotaxis. In particular, the quorum sensing is used to obtain the parameter information on bandwidth, delay, and error rate to facilitate the subsequent forwarding of packets; and the adaptive chemotaxis is used to determine the optimal interface for forwarding the packets through the information measurement. In addition, different from [6-9], this paper evaluates the proposed ICBQ based on real Netflix dataset rather than the random dataset, which guarantees the credibility and availability of ICBQ. In summary, the contributions of this paper have two aspects. On one hand, ICBQ simulates the behaviors of bacteria; on the other hand, ICBQ uses the real Netflix dataset.

The remainder of the paper is structured as follows. Section 2 reviews and analyzes the related work. Section 3 presents two behaviors of bacteria in CCN routing. In Section 4 , the interface forwarding strategy is introduced in detail. The experimental results are shown in Section 5. Finally, Section 6 concludes this paper and gives the future research directions.

\section{Related Work}

This section introduces existing works related to the routing strategy in CCN. Many investigations have been conducted on the routing strategy, employing some methods to address routing problems, which can be divided into three categories according to used methods: mathematical methods for routing, machine learning for routing, and intelligent algorithms for routing.

Some studies were carried out on routing based on mathematical methods. Paper [10] proposed a cache-aware social-based QoS routing method for routing, which built three kinds of social relationships to show the relationship among routers, namely, neighbors, interest friends, and response friends. Paper [11] proposed a gateway-aware congestion control method for ICN, where gateway provided a price for each user by evaluating the congestion situation related to the PIT information, and efficiently addressed congestion problem in routing. Paper [12] proposed an OST-based forwarding strategy based on network metrics, and experiments showed that proposed strategy performed best compared with other state-of-the-art methods in terms of latency and dissemination efficiency. Paper [13] proposed a Hash Routing based on the geographic information to address the locating problem in routing, which directly mapped the name of content to geographic information. Paper [14] proposed a probabilistic strategy to reduce the impact of the broadcast storm on interest forwarding in content-centric vehicular networks, where the forwarding probability is computed based on the neighbors' density of received interest. Reference [15] proposed two adaptive forwarding strategies, namely, Search and Discovery, to reduce the redundancy of the Interest and Data.

Machine learning has been applied to routing problems. Paper [16] proposed a novel adaptive forwarding strategy based on reinforcement learning with the random neural network to address interest forwarding, which used an online learning algorithm and reinforcement learning using the random neural network, to forward interest packets. Paper [17] designed and implemented IQ-Learning (Interest Q-Learning) strategy and DQ-Learning (Data Q-Learning) strategy to improve the efficiency and adaptivity of forwarding, which can make the best forwarding choice based on the past experience. Paper [18] proposed a novel adaptive forwarding scheme based on Q-learning to optimize the delivery latency in terms of forwarding characteristics of interest requests, which applied heuristic knowledge to the standard Q-learning algorithm.

Recently, some investigations have been conducted on using intelligent algorithms to address routing problems. Paper [19] mapped ant colony optimization into ICN and used ant colony foraging behavior to simulate the process of routing and proposed an ACO-inspired routing strategy supporting mobility to locate the content in routing. Paper [20] proposed a QoS-supported routing forwarding strategy based on ant colony optimization to achieve optimal routing strategy by choosing the route satisfying QoS requirement. Paper [21] proposed a greening domain and ant colony based joint forwarding method to optimize power consumption. In the paper, a packet forwarding method is used to support Quality of Service.

Existing routing strategies mentioned above can address routing problems to some extent, but there are some unsolved problems in these methods, such as dynamic change of network environment.

In this paper, we propose a Bacterial Quorum pattern inspired routing strategy for $\mathrm{CCN}$, which tries to simulate the behaviors of bacteria, including quorum sensing and adaptive chemotaxis. In the proposed strategy, the quorum sensing can obtain the parameter information related to bandwidth, delay, and error rate to promote packets forwarding; and the adaptive chemotaxis can choose the optimal interface to forward the packets based on the information evaluation.

\section{Behaviors of Bacteria in CCN Routing}

3.1. Thought Incubation. The bacteria are regarded as the simplest microorganism because of their easy description on the individual behavior, and they follow the principle of the optimal foraging theory. In particular, the bacteria have the outstanding adaptive ability in the complex environment [22], and they have been considered as the important research object from the perspective of biomicroorganism discipline. To be specific, the location during the process of bacteria foraging depends on the special chemotaxis behavior, including two movements modes, that is, swimming and flipping. When the environment includes the abundant resources, the bacteria keep on moving along the current 
direction; otherwise, when the resources in the environment are scarce, the bacteria also keep on moving but select a direction in a random way. The above two behaviors are called swimming and flipping, respectively. In terms of the flipping behavior, if the adaptation to the environment can be optimized gradually, then the bacteria keep on moving along the current direction until the degree of environment optimization cannot be improved. According to the special chemotaxis behavior, the bacteria always can keep on moving to the location that has the abundant resources, that is, adaptive chemotaxis.

In addition to the chemotaxis behavior, the bacteria also have the capability of drawing on advantages and avoiding disadvantages, that is, quorum sensing. Specifically, the multiple bacteria can share information and work in the collaborative way. Therein, each bacterium releases the induction signal to attract the other bacteria to move towards it. In addition, when some bacteria encounter the hazardous substance, they release the exclusive signal to enable the other bacteria to keep away from the hazardous substance.

According to the above statements, this paper plans to apply the behaviors of bacteria into $\mathrm{CCN}$ routing. To be specific, the bacteria have the adaptive and self-learning feature during the process of foraging, which can help improve the CCN routing efficiency. On the other hand, the behaviors, including adaptive chemotaxis and quorum sensing, represent the hop-by-hop mode, which can help obtain the network status during the process of CCN routing.

3.2. Adaptive Chemotaxis. In this paper, suppose that the chemotaxis direction of bacteria always depends on the awareness signal; that is to say, the bacteria keep on moving without the random way. During the process of adaptive chemotaxis, there are two kinds of bacteria, that is, interest bacteria and probing bacteria. The former are used to forward packets and the latter are used to collect the network status. In particular, each CCN node is equipped with three main elements, that is, Content Store (CS), Pending Interest Table (PIT), and Forwarding Information Base (FIB). The adaptive chemotaxis process (just like the interest routing) can be described as follows.

When CCN node receives bacteria, it first checks the CS. If the CS has the matched content entry, the following is to check whether the bacteria are the interest bacteria. If yes, the content is returned and the awareness signal is also returned to collect the network status along the current path; otherwise, only the awareness signal is returned and the packet is dropped (i.e., the bacteria are dead). On the other hand, if the CS has no matched content entry, the following is to check the PIT and see whether the PIT has the matched content name entry. If no, a new PIT entry is created including content name and the incoming interface of bacteria (interest bacteria are recorded in the interest interface table and probing bacteria are recorded in the probing interface table). If yes, the PIT is updated. Under such condition, if the bacteria are the interest bacteria, the behavior of chemotaxis is be continued; if they are the probing bacteria, only the incoming interface is added and the probing bacteria are dead.

The mathematical equation on the location updating of chemotaxis behavior is defined as follows:

$$
l(i, j+1)=l(i, j)+s(i) * \Delta * p,
$$

where $l(i, j)$ is the location after the $j$ chemotaxis behaviors with respect to bacteria $i, s(i)$ is the step size of swimming, $\Delta$ is the velocity vector, and $p$ is the regulator factor.

3.3. Quorum Sensing. Although the chemotaxis behavior of bacteria has an important impact on conducting the CCN routing, always there exists randomness in the corresponding process, which greatly decreases the efficiency of $\mathrm{CCN}$ routing. To this end, the quorum sensing can eliminate the nondeterminacy during the process of chemotaxis behavior. On this basis, the CCN node can compute the optimal transmission path of packets according to the carried parameters by the awareness signal (see Section 3.2 for details), and the optimal path is maintained and regarded as the empirical one for the subsequent content requests, which greatly increases the efficiency of $\mathrm{CCN}$ routing. The quorum sensing process (just like the data routing) can be described as follows.

When the content provider is found, the awareness signal is generated. After that, the awareness signal is sent via the recorded incoming interface in the PIT. When the CCN node receives the awareness signal, the PIT is firstly checked to see whether there is matched PIT entry. If no, the awareness signal is dropped directly. On the contrary, if there is matched PIT entry and the awareness signal is originated from the probing bacteria, the network status parameters of the transmission path are computed according to the carried information including bandwidth, delay, and error rate; at the same time, the other same awareness signal(s) is/are waited. However, if the received awareness signal is originated from the interest bacteria, the contents are returned to the interest requester via the interface to which the interest bacteria correspond and the related PIT entry will be deleted.

Let $b w_{i}$ denote the bandwidth of the traversed path $i$, let $b w_{j}$ denote the bandwidth of the traversing link $j$, let $\mathrm{d} l_{i}$ denote the corresponding delay of the traversed path $i$, let $\mathrm{d} l_{j}$ denote the corresponding delay of the traversing link $j$, let $e r_{i}$ denote the error rate of the traversed path $i$, and let $e r_{j}$ denote the error rate of the traversing link; and the related bandwidth, delay, and error rate after the awareness signal of probing bacteria traversing link $j$ are defined as follows: 


$$
\begin{aligned}
b w & =\min \left\{b w_{i}, b w_{j}\right\}, \\
\mathrm{d} l & =\min \left\{\mathrm{d} l_{i}, \mathrm{~d} l_{j}\right\}, \\
e r & =1-\left(1-e r_{i}\right) *\left(1-e r_{j}\right) .
\end{aligned}
$$

\section{Forwarding Strategy}

The bacteria can obtain the final foraging path based on two operations, that is, adaptive chemotaxis and quorum sensing. In the similar way, regarding the forwarding of interest packets, it is subject to several network status parameters, such as bandwidth, delay, and error rate. In fact, the optimal interface forwarding based on the multiple factors belongs to the multiattribute decision-making problem; this is a reason why the awareness signal of probing bacteria is explored (the collection of network status parameters). To be specific, such awareness signal provides support for these interest bacteria which request the same content, and thus the collaborative mode is formed.

In other words, the forwarding strategy based on interest bacteria strictly depends on bandwidth, delay, and error rate, and the optimal forwarding interface is obtained via the computation on them. In this paper, in order to manifest the objectivity of weight setting during the process of interface computation, we use the entropy weight method [23] to set the weights.

Suppose that there are $m$ forwarding interfaces in terms of some CCN node, and one matrix on the network status parameters can be constructed, that is, $R=\left(X_{i j}\right) m * 3$. Therein, the data elements in the first column represent the available bandwidth, those in the second column represent the corresponding delay, and those in the third column represent the corresponding error rate. Let $X_{i j}^{\prime}$ denote the standardized result of $X_{i j}$; we have

$$
\begin{aligned}
X_{i 1}^{\prime} & =\frac{\left(X_{i 1}-\min \left\{X_{i 1}\right\}\right)}{\left(\max \left\{X_{i 1}\right\}-\min \left\{X_{i 1}\right\}\right)}, \\
X_{i k}^{\prime} & =\frac{\left(\max \left\{X_{i k}\right\}-X_{i k}\right)}{\left(\max \left\{X_{i k}\right\}-\min \left\{X_{i k}\right\}\right)} .
\end{aligned}
$$

Furthermore, for the attribution $j$, let $e_{j}$ denote the entropy value of attribution $j$, and we have

$$
e_{j}=-t^{*} \sum_{i=1}^{m}\left(Q_{i j}-\ln Q_{i j}\right),
$$

where $t=(\ln m)-1$ and $Q_{i j}=\left(X_{i j}^{\prime} \sum_{i=1}^{m} X_{i j}^{\prime}\right)$.

Let $w_{j}$ denote the weight of one network status parameter, and we have

$$
w_{j}=\frac{\left(1-e_{j}\right)}{\sum_{j=1}^{3}\left(1-e_{j}\right)} .
$$

\section{Experimental Results}

The proposed ICBQ is implemented by Intel (R) Core (TM) i5-4590 CPU @ $3.46 \mathrm{GHz}$ and $8 \mathrm{~GB}$ RAM, and the programming language is $\mathrm{C}++$. In particular, the simulation is driven based on the real Netflix dataset [24] over the GTS network topology [25]. To be specific, the Netflix dataset includes 6512 users, 120000 requests, and 521 content providers. The GTS network topology includes 130 nodes and 168 links, as shown in Figure 1.

Besides, 6512 users, 120000 requests, and 521 content providers are distributed into the GTS network topology according the distribution laws. Furthermore, the used parameter $p$ is set as 0.5 , the number of bacteria for each round of iteration is set as 100, and the number of simulations is set as 30 . In addition, four metrics, that is, the average routing success rate, the average routing delay, the average load balance degree, and the average energy efficiency, are considered the criteria of evaluation performance. Moreover, the research works in $[7,8]$ are considered two baselines for comparison, because they are the latest intelligent $\mathrm{CCN}$ routing strategies, which are shortened as IJCS and ITL, respectively. Therein, [7] devises an energyefficient QoS routing by using the intelligent drops algorithm. Reference [8] proposes an intelligent Q-learning based on reinforcement learning to address the congestion control problem. In this paper, we send five groups of different interest requests, that is, $600,800,1200,1500$, and 2000 , and the 6100 interest requests are nonoverlapping.

5.1. Routing Success Rate. The routing success rate is defined as a ratio between the number of interest requests sent from users and that which can find the requested contents. The experimental results on the average routing success rate with respect to ICBQ, IJCS, and ITL under different interest requests are shown in Figure 2.

We find that the proposed ICBQ has the highest average routing success rate, and, especially at the last phase, the average routing success rate can approach $100 \%$. This suggests that the bacterial quorum inspired way can improve the CCN routing success rate. In addition, we also can find that the average routing success rate increases with increasing the number of sent interest requests; this is because the system gradually tends to the stable status. IJCS and ITL cannot obtain the satisfactory over GTS network topology because they cannot adjust the state in time. In particular, the path obtained by the intelligent drops algorithm is not perhaps optimal. Also, the reinforcement learning only guarantees the stability, while it cannot obtain the optimal routing success rate.

5.2. Routing Delay. The routing delay $(\mathrm{ms})$ is defined as a difference between the time point when the interest request is sent and that when the requested content is obtained or the failure signal is returned. The experimental results on the average routing delay with respect to ICBQ, IJCS, and ITL under different interest requests are shown in Figure 3.

We find that the proposed ICBQ has the smallest average routing delay and there are three main reasons. At first, the ICBQ uses the probing bacteria and saves the transmission delay. Then, the size of interest bacteria is very small, which can be transmitted in a fast speed. At last, the consumed time 


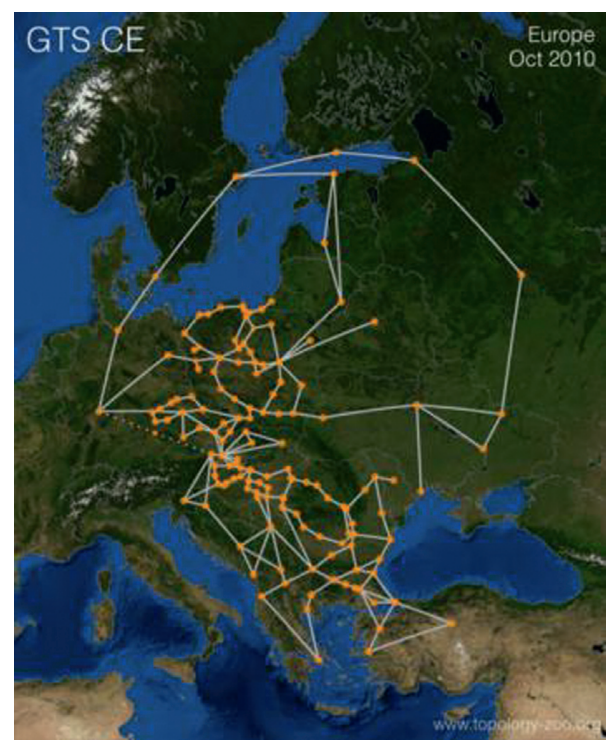

FIGURE 1: The GTS network topology used for simulation, including 130 nodes and 168 links.

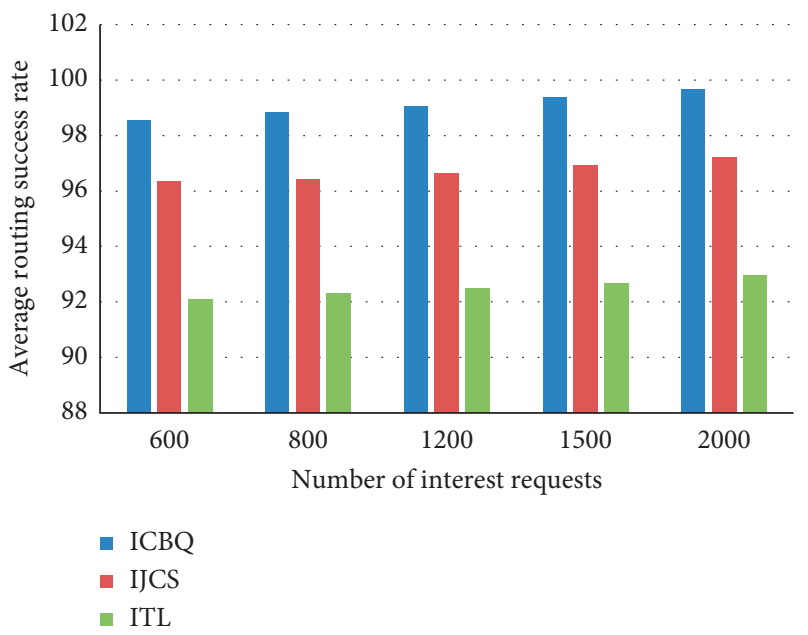

FIgURE 2: The average routing success rate (\%) among ICBQ, IJCS, and ITL.

by bacterial quorum is smaller than that by intelligent drops algorithm or that by reinforcement learning.

5.3. Load Balance Degree. The load balance degree is defined as a dispersion coefficient regarding all bandwidth values in the GTS network topology, and the small dispersion coefficient value indicates the good network performance. The experimental results on the average load balance degree with respect to ICBQ, IJCS, and ITL are shown in Figure 4.

We find that the proposed ICBQ has the best load balance degree, and this is because ICBQ fully considers the bandwidth factor. However, IJCS and ITL do not consider the bandwidth distribution; thus they have the relatively large load balance degree. In addition, the intelligent drops algorithm in IJCS usually converges to a path; thus the load is unbalanced. Similarly, ITL uses the reinforcement learning, which cannot balance the global network load; thus it cannot obtain the satisfactory load balance.

5.4. Energy Efficiency. The energy efficiency is a very important metric to measure whether the algorithm is green because ICT particularly pays attention to the energy saving. For details on the definition of energy efficiency, we refer the reader to [26]. The experimental results on the average energy efficiency with respect to ICBQ, IJCS, and ITL are shown in Figure 5.

We find that the proposed ICBQ has the highest energy efficiency, followed by IJCS and ITL, because ICBQ 


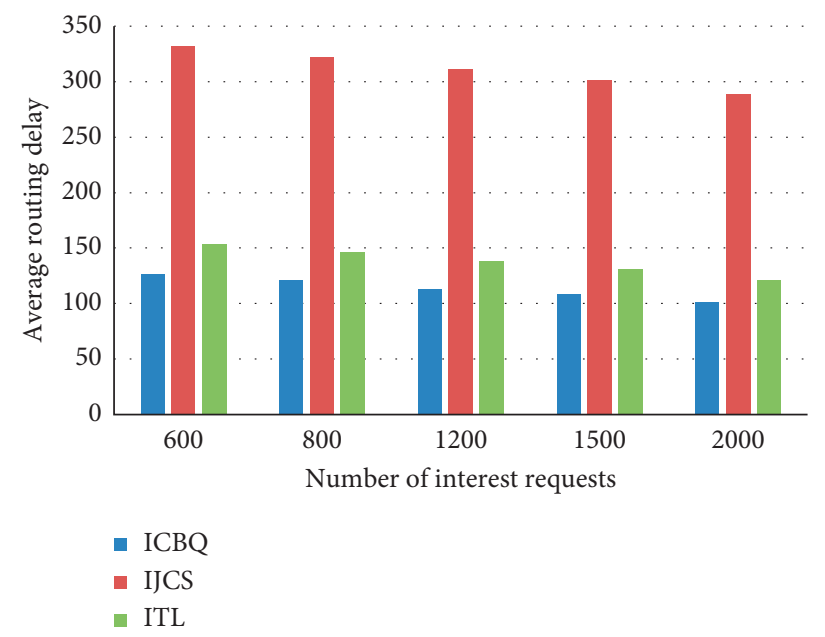

FIGURE 3: The average routing delay (ms) among ICBQ, IJCS, and ITL.

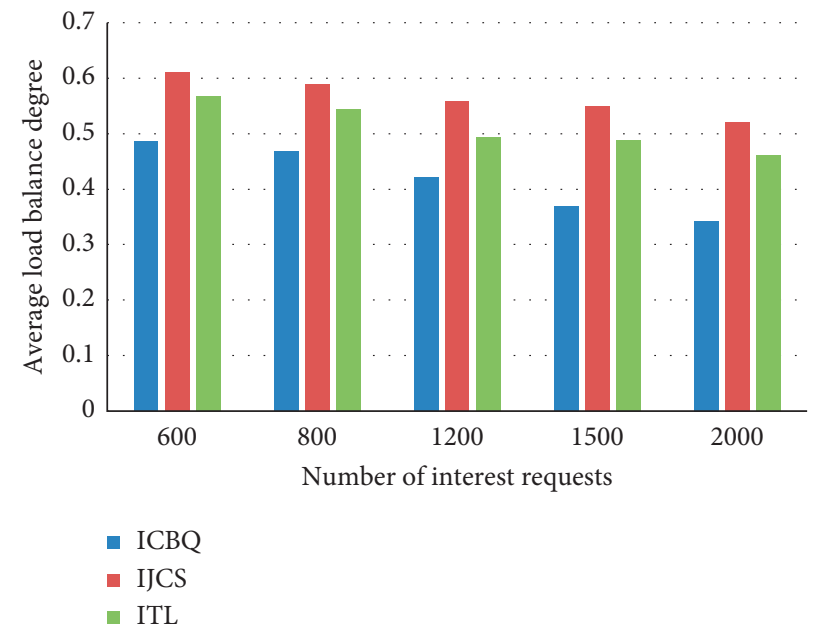

Figure 4: The average load balance degree among ICBQ, IJCS, and ITL.

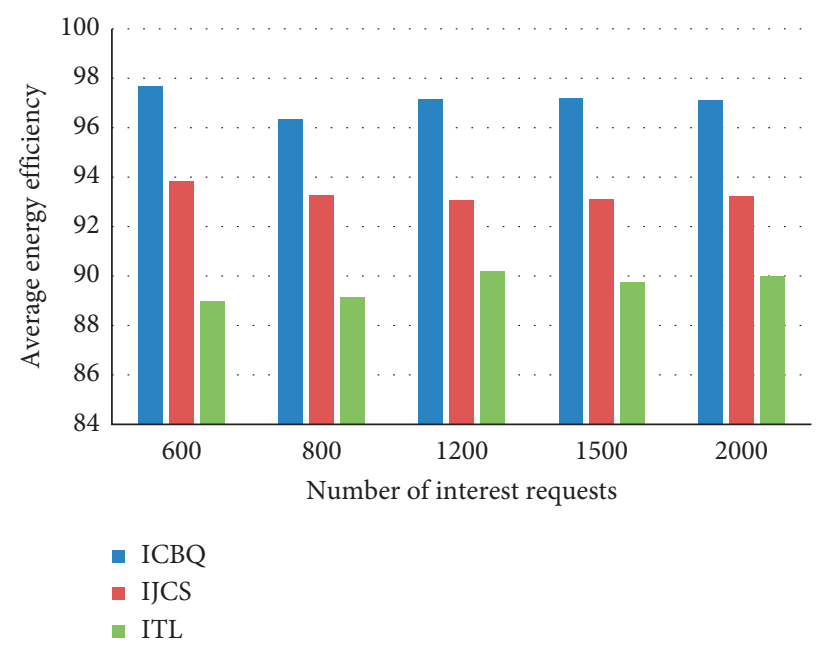

FIGURE 5: The average energy efficiency (\%) among ICBQ, IJCS, and ITL. 
consumes the smallest energy. For IJCS and ITL, they need the multiple iterations to complete the interest forwarding, which consume much more energy.

\section{Conclusions}

This paper investigates the intelligent $\mathrm{CCN}$ routing based on the behaviors of bacteria. At first, two behaviors of bacteria are adaptive chemotaxis and quorum sensing. Then, a CCN routing strategy based on the distributed hop-by-bop way is introduced, in which interest bacteria and probing bacteria are involved. Finally, the simulation is made based on the real dataset over the real network topology, and the experimental results demonstrate its feasibility and efficiency by testing routing success rate, routing delay, load balance degree, and energy efficiency. In the future, we plan to improve the forwarding strategy and discuss the convergence of bacterial quorum. In addition, we also plan to make the large-scale experiments at the testbed.

\section{Data Availability}

The network topology data used to support the findings of this study have been deposited at http://www.topology-zoo. org.

\section{Conflicts of Interest}

The authors declare that they have no conflicts of interest.

\section{References}

[1] H. Li, G. Shou, Y. Hu et al., "Mobile edge computing: progress and challenges," in Proceedings of the IEEE International Conference on Mobile Cloud Computing Services, and Engineering (Mobile Cloud), pp. 83-84, Oxford, UK, March 2016.

[2] P. T. Dat, A. Kanno, N. Yamamoto, and T. Kawanishi, "5G transport networks: the need for new technologies and standards," IEEE Communications Magazine, vol. 54, no. 9, pp. 18-26, 2016.

[3] Y. Liu, K. Wang, K. Qian et al., “Tornado: enabling blockchain in heterogeneous Internet of things through a space-structured approach," IEEE Internet of Things Journal, vol. 7, no. 2, pp. 1273-1286, 2019.

[4] Cisco Annual Internet Report (2018-2023) White Paper, https://www.cisco.com/c/en/us.

[5] G. Xylomenos, C. N. Ververidis, V. A. Siris et al., "A survey of information-centric networking research," IEEE Communications Surveys \& Tutorials, vol. 16, no. 2, pp. 1024-1049, 2014.

[6] R. Hou, Y. Chang, and L. Yang, "Multi-constrained QoS routing based on PSO for named data networking," IET Communications, vol. 11, no. 8, pp. 1251-1255, 2017.

[7] L. Zhang and J. Lv, "Intelligent information centric networking routing scheme under imprecise information," International Journal of Communication Systems, vol. 32, no. 4, pp. 1-20, 2019.

[8] W. Meng and L. Zhang, "Q-learning-based congestion control strategy for information-centric networking," Internet Technology Letters, 2020, In press.

[9] J. Lv, X. Wang, K. Ren, M. Huang, and K. Li, "ACO-inspired information-centric networking routing mechanism," Computer Networks, vol. 126, pp. 200-217, 2017.
[10] D. Qu, X. Wang, M. Huang, K. Li, S. K. Das, and S. Wu, “A cache-aware social-based QoS routing scheme in Information Centric Networks," Journal of Network and Computer Applications, vol. 121, no. 7, pp. 20-32, 2018.

[11] W. Li, S. Wang, Y. Xu, and S. Lu, "Charging on the route: an online pricing gateway congestion control for ICNs," IEEE Transactions on Network and Service Management, vol. 17, no. 1, pp. 239-250, 2020.

[12] C. Borrego, M. Amadeo, A. Molinaro et al., "Forwarding in opportunistic information-centric networks: an optimal stopping approach," IEEE Communications Magazine, vol. 58, no. $5,2020$.

[13] H. Jung and S. Kim, "A Multiple Hash Routing Scheme for Fast Data Retrieval in ICN," in Proceedings of the 2018 International Conference on Information and Communication Technology Convergence (ICTC), Jeju, South Korea, October 2018.

[14] R. Tizvar and M. Abbaspour, "A density-aware probabilistic interest forwarding method for content-centric vehicular networks," Vehicular Communications, vol. 23, Article ID 100216, 2019.

[15] S. Iqbal, "Adaptive for-warding strategies to reduce redundant Interests and Data in named data net-works," Journal of Network and Computer Applications, vol. 106, pp. 33-47, 2018.

[16] O. Akinwande, "Interest forwarding in named data networking using reinforcement learning," Sensors, vol. 18, no. 10, Article ID 3354, 2018.

[17] B. Fu, L. Qian, Y. Zhu et al., "Reinforcement learning-based algorithm for efficient and adaptive forwarding in named data networking," in Proceedings of the 2017 IEEE/CIC Inter-national Conference on Communica-tions in China (ICCC), Qingdao, China, October 2017.

[18] M. Zhang, X. Wang, T. Liu, J. Zhu, and Q. Wu, "AFSndn: a novel adaptive forwarding strategy in named data networking based on Q-learning," Peer-to-Peer Networking and Applications, vol. 13, no. 4, pp. 1176-1184, 2020.

[19] J. Lv, X. Wang, and M. Huang, "ACO-inspired ICN routing mechanism with mobility support," Applied Soft Computing, vol. 58, pp. 427-440, 2017.

[20] Q. Huang and F. Luo, "Ant-colony optimization based QoS routing in named data networking," Journal of Computational Methods in Sciences \& Engineering, vol. 16, no. 3, pp. 671-682, 2016.

[21] C. Li, W. Liu, L. Wang et al., "Energy-efficient quality of service aware for-warding scheme for Content-Centric Networking," Journal of Network and Computer Applications, vol. 58, pp. 241-254, 2015.

[22] K. M. Passino, "Biomimicry of bacterial foraging for distributed optimization and control," IEEE Control Systems, vol. 22, no. 3, pp. 52-67, 2002.

[23] Y.-M. Wang and Y. Luo, "Integration of correlations with standard deviations for determining attribute weights in multiple attribute decision making," Mathematical and Computer Modelling, vol. 51, no. 1-2, pp. 1-12, 2010.

[24] Netflix Dataset, https://pan.baidu.com/s/1dDtmbW9.

[25] GTS Network Topology, http://www.topology-zoo.org.

[26] X. Wang, J. Lv, M. Huang, K. Li, J. Li, and K. Ren, "Energyefficient ICN routing mechanism with QoS support," Computer Networks, vol. 131, pp. 38-51, 2018. 